\title{
The effects of functional electrical stimulation applied to the gluteus medius and tibialis anterior on stair climbing ability in persons with stroke
}

\author{
Jewon Jung ${ }^{a}$, Yijung Chung ${ }^{b}$ \\ aDepartment of Physical Therapy, The Graduate School, Sahmyook University, Seoul, Republic of Korea \\ ${ }^{b}$ Department of Physical Therapy, College of Health Science and Social Welfare, Sahmyook University, Seoul, Republic of Korea
}

\begin{abstract}
Objective: The aim of this research was to investigate the effects of functional electrical stimulation (FES) applied to the gluteus medius (Gm) and tibialis anterior (TA) during stair climbing in persons with stroke compared to FES applied to the TA only during stair climbing, and during stair climbing without FES in persons with stroke.
\end{abstract}

Design: Cross-sectional study.

Methods: Twenty subjects with stroke participated in this study. Subjects were included if: 1) they were diagnosed as stroke at least 6 months before; 2) had Mini Mental State Examination- Korean score of 24 or higher; 3) were able to climb a flight of 10 stairs independently (with or without walking aid). The patients walked 10 stairs 3 times with FES applied to the Gm and TA, only TA, or no FES. There was a 1-minute rest period between each bout. The assessments were made using the Timed Up \& Down Test and the Wii Balance Board.

Results: Stair climbing with FES applied to the Gm and TA was significantly faster than stair climbing with FES applied to the TA only and without FES $(p<0.05)$. Stair climbing with FES applied to the Gm and TA exhibited significantly greater sway velocity than stair climbing without FES $(p<0.05)$. However, maximal sway distances were not significantly different between groups. Conclusions: Stair climbing with FES applied to the Gm and TA can be an important component of a rehabilitation program for improving stair climbing ability in persons with stroke.

Key Words: Electrical stimulation, Postural balance, Stair climbing, Stroke

\section{Introduction}

Abnormal walking can occur if there is excessive asymmetry or angle increases of the knees during walking. An example of when this can occur is after a stroke incident [1]. Postural instability or fluctuations in persons with stroke are more likely to occur when there is an increased discrepancy in lower extremity strength [2]. In addition, the pelvis tends to exhibit more instability in those affected by stroke compared to those who are unaffected, which resembles the movement of an inverted pendulum [3]. In the case of older adults, trunk instability is severe, especially in those who perform unusual gait [4]. For patients with central nervous system injuries, restoration of the patient's gait is an integral part of rehabilitation and decides whether if the person will be able to return to their homes or the society [5]. Gait recovery has been an important goal in the rehabilitation of stroke survivors for a long time, and significant time and effort is being spent to reach this goal. In recent years, participation in social activities besides simple walking is regarded as the ultimate goal of rehabilitation [6]. Stairs are frequently encountered in everyday life, but walking on stairs is different

Received: 24 August, 2018 Revised: 11 September, 2018 Accepted: 13 September, 2018

Corresponding author: Yijung Chung (ORCID https://orcid.org/0000-0002-2431-8895)

Department of Physical Therapy, College of Health Science and Social Welfare, Sahmyook University, 815 Hwarang-ro, Nowon-gu, Seoul 01795 , Republic of Korea

Tel: 82-2-3399-1637 Fax: 82-2-3399-1639 E-mail: yijung36@syu.ac.kr

(c) This is an Open-Access article distributed under the terms of the Creative Commons Attribution Non-Commercial License (http://creativecommons.org/licenses/ by-nc/4.0) which permits unrestricted non-commercial use, distribution, and reproduction in any medium, provided the original work is properly cited.

Copyright $\odot 2018$ Korean Academy of Physical Therapy Rehabilitation Science 
from walking on flat surfaces [7]. Compared to walking on flat surfaces, stair climbing requires more energy and lower extremity strength due to the fact that horizontal and vertical movements occur together while maintaining balance [8]. Stairway walking ability can be a measure of whether a patient can return to the community [9].

In addition to the gluteus maximus, the gluteus medius (Gm) has increased muscular activity during fast gait, weight loading, and early to mid-stance phase. During stair-climbing, the Gm increases muscle activity from the time of loading until prior to the start of the swing phase [10]. Appropriate contraction of the Gm muscle not only affects trunk stability but also affects the timing of the stance phase of the opposite leg [11]. With Gm weakness, the width between the lower extremity increases in order to compensate for the stability that has been lost during walking [12]. When the body is tilted to the right or to the left, the muscular activity of the $\mathrm{Gm}$ and the tibialis muscle is increased in normal persons but decreased in persons with stroke [13,14]. The muscular activity of the Gm was higher when walking up the stairs sideways compared to forwards [15].

Functional electrical stimulation (FES) is a successful complement to foot drop during walking in persons with chronic stroke [16]. By supplementing the foot drop, excessive knee flexion can be prevented at the initial swing phase [17]. However, Tan et al. [16] reported that applying FES to the ankle alone cannot compensate for problems occurring in the hip. In functional training, it is more effective to apply FES to the anterior tibialis and the Gm muscle rather than the anterior tibialis muscle alone during gait training [18]. Using FES as a basic principle, robot therapy can be used for gait training purposes on flat ground as well as for moving up and down stairs and avoiding obstacles [5]. Studies show that asymmetry leads to instability, but studies showing that reducing asymmetry can lead to increased stability are also in need [19].

Therefore, this study investigated the time it takes for persons with stroke to go up and down the stairs, how the center of gravity of the trunk moves, and whether there are quantitative or qualitative changes when applying FES to the Gm and tibialis anterior (TA).

\section{Methods}

\section{Subjects}

Twenty patients who were receiving treatment form the Guri K rehabilitation clinic in Gyeonggi-do were eligible for
Table 1. The general and medical characteristics of subjects

$(\mathrm{N}=20)$

\begin{tabular}{lc}
\hline \multicolumn{1}{c}{ Variable } & Subjects \\
\hline Sex (male/female) & $17 / 3$ \\
Age (y) & $54.05(8.30)$ \\
Onset Causes (cerebral infarction/ & $11 / 9$ \\
$\quad$ cerebral hemorrhage) & \\
Paralysis Site (left/right) & $10 / 10$ \\
MMSE-K(points) & $27.05(2.31)$ \\
\hline
\end{tabular}

Values are presented as number only or mean (SD).

MMSE-K: Mini Mental State Examination-Korea.

the study (Table 1). Stroke participants with a Korean version of the Mini Mental State Examination-Korea score of at least 24 points, those who can follow instructions, and those who can go up and down 10 steps with or without an assistive device were included in the study [20]. Those who had problems with language skills as well as lack of spatio-temporal and visual field senses, which are needed to walk the stairs, were excluded from the experiment [21]. This study was conducted with the consent of the Bioethics Review Committee of Sahmyook University (IRB No. 2-1040781AB-N2016019HR).

Subjects were divided into either the FES applied to the gluteus medius and tibialis anterior (GmTA), FES applied to the TA only, or the general (Ge) group with no FES. Subjects provided their informed consent prior to participating in the study.

\section{Measurements}

\section{Timed Up \& Down Test}

Before going up and down the stairway, subjects were informed to 'go up and down safely at a comfortable pace'. After giving the start signal, one evaluator measured the time. Before the start signal, the subjects maintained their feet from falling off the ground and waited for the signal. After the start signal, the subject went up and down 10 steps and once both feet touched together on the ground, the timer was stopped. The measurement unit was set to $1 / 100$ seconds. Although the subjects were allowed to rest during the stair activity, the timer was not stopped. At this time, if necessary, subjects were allowed to use the rail [20]. The Timed Up \& Down Test (TUDT) has a reliability of 0.98 and a high correlation $(|r|=0.80-0.90)$ compared to the usual walking ability measurement tools, such as Timed Up \& Go and the 6-Minute Walk test [22]. 


\section{Wii Balance Board}

The Wii Balance Board (Nintendo, Japan) was used to assess the center of gravity of the subjects during stair walking. The Wii Balance Board was placed on the last step of the staircase and subjects were given a demonstration on how to walk the stairs. Prior to stair climbing, the subjects were not particularly told to step on the Wii Balance Board and pass to go on stairs. None of the subjects walked the stairs without stepping on the Wii Balance Board. The stair walking of each subject was repeated under the same condition. The Wii Balance Board was connected to the laptop with Bluetooth connection. The movement of the center of gravity of the subjects was synthesized by the information sensed by the pressure sensors at each corner of the Wii Balance Board. The data extraction rate was set at $100 \mathrm{~Hz}$ and filtered at $10 \mathrm{~Hz}$. The center of gravity information extracted from the subject was processed through Microsoft Excel 2007 (Microsoft, Redmond, WA, USA). The inter-instrument reliability of the above measurement tools is 0.83-0.99 [23]. The maximal sway distance (MSD) is defined as the difference between the minimum and maximum values of the $\mathrm{X}$-axis in the extracted values [24], and the sway velocity (SV) is defined as the speed of the $\mathrm{x}$-axis because each axis's velocity is representative of the resulting velocity [25].

\section{Statistical analysis}

All statistical analyses were performed using IBM SPSS Statistics ver. 19.0 software (IBM Co., Armonk, NY, USA) and descriptive statistics were used to describe the general characteristics of the subjects. The one-way repeated measures ANOVA was used to analyze the differences between the FES applied to the Gm and TA condition, FES applied only to the TA condition, and the no FES condition. The least significant difference was used for post hoc analyses, and the statistical significance level of all data was set at $p<0.05$.

Table 2. TUDT results for each condition

$(\mathrm{N}=20)$

\begin{tabular}{cccc}
\hline Variable & GmTA condition & TA condition & Ge condition \\
\hline TUDT (s) & $37.59(14.07)^{* * * *} 38.99(14.96)$ & $39.65(14.29)$ \\
\hline
\end{tabular}

Values are presented as mean (SD).

TUDT: Timed Up \& Down Test, GmTA: gluteus medius and tibialis anterior, TA: tibialis anterior, Ge: general.

*Statistically significant different from Ge $(p<0.05)$.

$* *$ Significantly different from TA $(p<0.05)$.

\section{Results}

\section{Timed Up \& Down Test}

The GmTA group was able to go up and down the stairs in a significantly shorter amount of time compared to the TA group or Ge group $(p<0.05)$. The results are as follows (Table 2).

\section{Wii Balance Board}

The SV and MSD measured by the Wii Balance Board are presented in Table 3.

\section{Sway velocity}

The GmTA group showed a significant increase in SV $(p<0.05)$ compared to the Ge group. There was no significant difference in the SV between the TA group and Ge group.

\section{Maximal Sway Distance}

There was no significant difference in MSD between the GmTA, TA, and the GE groups.

\section{Discussion}

This study investigated how the ability of persons with stroke to perform stair climbing was affected by applying FES. Walking speeds and sway velocities were increased during stair climbing when FES was applied to the Gm and anterior tibialis muscles. This is similar to the results of previous studies in which FES was applied to the Gm and anterior tibialis muscles of persons with stroke [26]. There was no significant difference in the MSD between groups in this study.

Based on the joint strategy (moving from the ankle to the hip joint) used to maintain balance [3], it was hypothesized that the degree of instability would decrease and the rate of instability would increase. This is because it was anticipated

Table 3. Comparison of SV and MSD for each condition (N=20)

\begin{tabular}{lccc}
\hline Variable & GmTA condition & TA condition & Ge condition \\
\hline $\mathrm{SV}(\mathrm{cm} / \mathrm{s})$ & $5.53(3.46)^{*}$ & $5.05(4.30)$ & $4.12(3.29)$ \\
$\mathrm{MSD}(\mathrm{s})$ & $7.74(2.43)$ & $7.12(1.78)$ & $7.08(1.58)$ \\
\hline
\end{tabular}

Values are presented as mean (SD).

SV: sway velocity, MSD: maximal sway distance, GmTA: gluteus medius and tibialis anterior, TA: tibialis anterior, Ge: general.

* Statistically significant different from Ge $(p<0.05)$. 
that the FES applied to the Gm and anterior tibialis muscle would assist with balance during walking. The experimental results showed that the rate of instability was higher than that of groups that did not have FES applied to the Gm and TA, but there was no significant difference in the degree of instability between the groups. A study by Marigold and Eng [27] showed that the greater the asymmetry of weight support, and that although the rate of instability increased, it did not increase as much as anticipated. Initially it was expected that the asymmetry of weight support would be reduced, which would increase the speed during stair- more quickly. In this study, the time required for stair walking was significantly different but there was no significant difference in asymmetry of weight support. Repetition of the experiment is required to assess the degree of left-right instability.

Compared with previous studies in which the gait speed was increased in persons with stroke when FES was applied to the Gm and TA [28,29], it was anticipated that stair climbing speed would also increase with the same intervention. Therefore, in future studies, it is necessary to reaffirm the effect of FES on the Gm and TA muscles on left-right instability during stair climbing in persons with stroke. With stair climbing training, it is necessary to investigate the changes in the stair climbing speeds with FES applied to the $\mathrm{Gm}$ and anterior tibialis.

\section{Conflict of Interest}

The authors declared no potential conflicts of interest with respect to the authorship and/or publication of this article.

\section{References}

1. Handžić I, Reed KB. Perception of gait patterns that deviate from normal and symmetric biped locomotion. Front Psychol 2015;6:199.

2. Kim MK, Kim SG. Analysis of the correlation between the differences in muscle strengths between the bilateral lower extremities and postural stability in healthy adults. J Phys Ther Sci 2015;27:3103-4.

3. Dickstein R, Abulaffio N. Postural sway of the affected and nonaffected pelvis and leg in stance of hemiparetic patients. Arch Phys Med Rehabil 2000;81:364-7.

4. Lee SW, Verghese J, Holtzer R, Mahoney JR, Oh-Park M. Trunk sway during walking among older adults: norms and correlation with gait velocity. Gait Posture 2014;40:676-81.

5. Schmidt H, Werner C, Bernhardt R, Hesse S, Krüger J. Gait rehabilitation machines based on programmable footplates. J Neuroeng Rehabil 2007;4:2.

6. Lord SE, McPherson K, McNaughton HK, Rochester L, Weatherall
M. Community ambulation after stroke: how important and obtainable is it and what measures appear predictive? Arch Phys Med Rehabil 2004;85:234-9.

7. Seo K, Kim J, Wi G. The effects of stair gait exercise on static balance ability of stroke patients. J Phys Ther Sci 2014;26:1835-8.

8. Park KH, Kim DY, Kim TH. The effect of step climbing exercise on balance and step length in chronic stroke patients. J Phys Ther Sci 2015;27:3515-8

9. Alzahrani MA, Dean CM, Ada L. Ability to negotiate stairs predicts free-living physical activity in community-dwelling people with stroke: an observational study. Aust J Physiother 2009;55: 277-81.

10. Lyons K, Perry J, Gronley JK, Barnes L, Antonelli D. Timing and relative intensity of hip extensor and abductor muscle action during level and stair ambulation An EMG study. Phys Ther 1983; 63:1597-605.

11. Hase K, Stein RB. Turning strategies during human walking. J Neurophysiol 1999;81:2914-22.

12. Kubinski SN, McQueen CA, Sittloh KA, Dean JC. Walking with wider steps increases stance phase gluteus medius activity. Gait Posture 2015;41:130-5.

13. Duval K, Luttin K, Lam T. Neuromuscular strategies in the paretic leg during curved walking in individuals post-stroke. J Neurophysiol 2011;106:280-90.

14. Maeda Y, Tanaka T, Nakajima Y, Shimizu K. Analysis of postural adjustment responses to perturbation stimulus by surface tilts in the feet-together position. J Med Biol Eng 2010;31:301-5.

15. Mercer VS, Gross MT, Sharma S, Weeks E. Comparison of gluteus medius muscle electromyographic activity during forward and lateral step-up exercises in older adults. Phys Ther 2009;89: 1205-14.

16. Tan Z, Liu H, Yan T, Jin D, He X, Zheng X, et al. The effectiveness of functional electrical stimulation based on a normal gait pattern on subjects with early stroke: a randomized controlled trial. Biomed Res Int 2014 Jul 10. doi: 10.1155/2014/545408. [Epub ahead of print].

17. Kesar TM, Perumal R, Jancosko A, Reisman DS, Rudolph KS, Higginson JS, et al. Novel patterns of functional electrical stimulation have an immediate effect on dorsiflexor muscle function during gait for people poststroke. Phys Ther 2010;90:55-66.

18. Mun BM, Kim TH, Lee JH, Lim JY, Seo DK, Lee DJ. Comparison of gait aspects according to FES stimulation position applied to stroke patients. J Phys Ther Sci 2014;26:563-6.

19. Kamphuis JF, de Kam D, Geurts AC, Weerdesteyn V. Is weightbearing asymmetry associated with postural instability after stroke? a systematic review. Stroke Res Treat 2013 Apr 28. doi: 10.1155/2013/692137. [Epub ahead of print]

20. Ng SS, Ng HH, Chan KM, Lai JC, To AK, Yeung CW. Reliability of the 12-step ascend and descend test and its correlation with motor function in people with chronic stroke. J Rehabil Med 2013;45:123-9.

21. Kirker SG, Simpson DS, Jenner JR, Wing AM. Stepping before standing: hip muscle function in stepping and standing balance after stroke. J Neurol Neurosurg Psychiatry 2000;68:458-64.

22. Flansbjer UB, Holmbäck AM, Downham D, Patten C, Lexell J. Reliability of gait performance tests in men and women with hemiparesis after stroke. J Rehabil Med 2005;37:75-82.

23. Abujaber S, Gillispie G, Marmon A, Zeni J Jr. Validity of the 
Nintendo Wii Balance Board to assess weight bearing asymmetry during sit-to-stand and return-to-sit task. Gait Posture 2015; 41:676-82.

24. Cote KP, Brunet ME, Gansneder BM, Shultz SJ. Effects of pronated and supinated foot postures on static and dynamic postural stability. J Athl Train 2005;40:41-6.

25. Mei Z, Zhao G, Ivanov K, Guo Y, Zhu Q, Zhou Y, et al. Sample entropy characteristics of movement for four foot types based on plantar centre of pressure during stance phase. Biomed Eng Online 2013;12:101.

26. Kim JH, Chung Y, Kim Y, Hwang S. Functional electrical stimulation applied to gluteus medius and tibialis anterior corre- sponding gait cycle for stroke. Gait Posture 2012;36:65-7.

27. Marigold DS, Eng JJ. The relationship of asymmetric weightbearing with postural sway and visual reliance in stroke. Gait Posture 2006;23:249-55.

28. Chung Y, Kim JH, Cha Y, Hwang S. Therapeutic effect of functional electrical stimulation-triggered gait training corresponding gait cycle for stroke. Gait Posture 2014;40:471-5.

29. Cho MK, Kim JH, ChungY, Hwang S. Treadmill gait training combined with functional electrical stimulation on hip abductor and ankle dorsiflexor muscles for chronic hemiparesis. Gait Posture 2015;42:73-8. 